\title{
Use of dynamic light scattering for assessing acute pain
}

Suzanne Broens, Adi Schejter Bar-Noam, Ilya Fine, Louis Shenkman, Monique van Velzen, et al.

Suzanne Broens, Adi Schejter Bar-Noam, llya Fine, Louis Shenkman, Monique van Velzen, Marieke Niesters, Albert Dahan, "Use of dynamic light scattering for assessing acute pain," Proc. SPIE 11075, Novel Biophotonics Techniques and Applications V, 110750N (22 July 2019); doi: $10.1117 / 12.2524523$

SPIE. Event: European Conferences on Biomedical Optics, 2019, Munich, Germany 


\title{
Use of dynamic light scattering for assessing acute pain
}

\author{
Suzanne Broens, ${ }^{\mathrm{a}}$ Adi Schejter Bar-Noam, ${ }^{\mathrm{b}}$ Ilya Fine, ${ }^{\mathrm{b},}$ Louis Shenkman, ${ }^{\mathrm{c}}$ Monique van Velzen, ${ }^{\mathrm{a}}$ \\ Marieke Niesters, ${ }^{\mathrm{a}}$ Albert Dahan ${ }^{\mathrm{a}}$ \\ ${ }^{a}$ Department of Anesthesiology, Leiden University Medical Center, Leiden, The Netherlands \\ ${ }^{b}$ Elfi-Tech Ltd., Science Park, Rehovot, Israel \\ ${ }^{\mathrm{c}}$ Tel Aviv University, Sackler School of Medicine, Tel Aviv, Israel
}

\begin{abstract}
Currently there is no accurate objective measure for monitoring pain during the state of drug-induced unconsciousness (such as during surgical anesthesia). Moreover, the absence of an objective measure for detecting pain hampers the physician's ability to provide an optimal dose of analgesics. We have developed a novel method for detecting pain by quantifying skin blood flow dynamics using a miniaturized dynamic light scattering (mDLS) sensor placed on the skin. Healthy awake volunteers were studied with mDLS sensors placed on both index fingers while being subjected to a series of cutaneous painful stimuli (electric shock and heat), randomly applied in a range between the subjects' pain threshold and tolerance. Power spectrum analysis of the recorded signal was performed with a focus on two frequency bands, representing relative blood flow of non-pulsatile vessels and larger pulsatile arterioles. Relative blood flow of pulsatile vessels decreased while flow of non-pulsatile vessels increased in response to painful stimulation, with a high correlation between the responses obtained on the right and left index fingers. The changes in hemodynamics that occur during painful stimulation suggest a redistribution of blood flow between pulsatile and non-pulsatile vessels, probably related to central activation of the sympathetic system combined with local dynamic autoregulatory responses. Thus, optical parameters of skin blood flow can detect nociceptive stimuli and consequently can serve as objective biomarkers of pain.
\end{abstract}

Keywords: pain, nociception, dynamic light scattering, laser Doppler, peripheral vessels, anesthesia, blood flow

*Contact Author, E-mail: ilyafine@elfi-tech.com

\section{INTRODUCTION}

\subsection{Background}

During the state of drug-induced unconsciousness (for example anesthesia or deep sedation), detection of nociceptive stimuli, such as surgical stimuli that cause tissue damage, relies commonly on the measurement of blood pressure or heart rate (HR). ${ }^{1}$ These measurements, however, may not detect all events or detect events with some delay. Recently, new non-invasive technologies have been developed to detect nociceptive events in awake and anesthetized individuals that rely on signals of the autonomic nervous system such as heart rate variability, pulse pressure, pupil diameter, peripheral vasoconstriction, skin galvanic response, or on a combination of these signals. ${ }^{1-5}$ Most studies indicate that nociceptive indices based on these autonomic signals outperform BP and HR in the detection of nociceptive events. Changes in blood flow could offer an additional option for detecting noxious responses during anesthesia, as is demonstrated with the Surgical Pleth Index (SPI) method. ${ }^{6}$ This method relies on heart rate variability (HRV) and total blood perfusion in the fingertip extracted from the plethysmographic signal.

We propose a new method for detection of nociceptive events by quantifying skin blood flow dynamics using a miniaturized dynamic light scattering (mDLS) sensor. ${ }^{8-9}$ This sensor enables extraction of multiple hemodynamic parameters that can indicate changes in the autonomic nervous system.

Novel Biophotonics Techniques and Applications V, edited by Arjen Amelink, Seemantini K. Nadkarni, Proc. of SPIE-OSA Vol. 11075, 110750N · C 2019 SPIE-OSA · CCC code: 1605-7422/19/\$21 · doi: 10.1117/12.2524523 


\subsection{Theoretical background}

The sensor technology used in the following experiments relies on a phenomenon known as dynamic light scattering. ${ }^{7}$ The laser beam from a miniaturized DLS probe is projected onto the skin, and the light scattered from the flowing RBCs in the blood vessels creates a speckle pattern on the mDLS detector. The overall measured dynamic light scattering pattern is originated by the interaction between the coherent light that is scattered by the moving red blood cells. The relative movement of the particles, which is responsible for the speckle dynamics, is characterized by the velocity shear rate. Therefore, for laminar flow, the signal measured by the mDLS sensor is correlated with the gradient of the velocity in the blood stream, also known as the shear rate $\gamma .{ }^{7}$

In a very simplified case, for the vessel of radius $R$, axis symmetric velocity profiles $v(r, t)$ can be described in cylindrical coordinates by this empirical relationship:

$$
v(r, t)=v(0)\left[1-\left(\frac{r}{R}\right)^{\xi}\right] f(t) \quad-R \leq r \leq R ;
$$

Where $v(0)$ - is maximum velocity at $\mathrm{r}=0$ and $\mathrm{R}$ is the radius of the vessel, $f(t)$ is a periodic function of heart beat frequency, and $\xi$ represents the degree of blunting. The velocity shear rate can be determined by:

$$
\gamma=\frac{\partial \dot{v}(r, t)}{\partial r}=\xi \cdot v(o, t) \cdot \frac{r^{\xi-1}}{R \xi} \quad, \quad v(o, t)=\frac{\xi+2}{\xi}<v(t)
$$

For each sub-ensemble $s$, the autocorrelation function decay $\mathrm{g}(\mathrm{s}, \tau)$ is given by:

$$
g(s, i)=\alpha * \exp \left[-\Gamma(s) \tau^{2}\right], \quad \Gamma(s)=\left(\gamma(s) d^{*} q\right)^{2}
$$

where $q=2 \cdot k \cdot \sin (\theta / 2), \theta$ - is scattering angle, $\mathrm{k}$ is wavelength number and $\mathrm{d}^{*}$ is the effective distance across the scattering volume in the direction of the velocity gradient. Since the skin is characterized by a variate of different vessels with different shear rates the overall autocorrelation function of the measured signal can be represented as a sum of $n$ weighted (w) contributions from different sub-ensembles of RBC's, corresponding to their shear rate:

$$
G(\tau)=\sum_{s=1}^{n} w(s) g(s, \tau)
$$

The power spectrum representation of this expression will be given by Fourier transform of

$$
\mathrm{P}(\omega)=w(i) \sum_{s=1}^{n} \int_{-\infty}^{\infty} g(s, \tau) \exp (i \omega \tau) d \tau
$$

Therefore, the total power spectrum can be represented as a sum of different bandpass, where each bandpass corresponds to the different shear rate RBCs. Thus, it is possible to extract multiple physiological parameters from this signal by analyzing the power spectrum of the signal, $\mathrm{P}(\omega, t)$, over time. ${ }^{8}$ We defined the hemodynamic index (HI) which corresponds to a certain range of shear rates determined by the frequencies $\omega_{1}$ and $\omega_{2}$ :

$$
H I\left[\omega_{1}, \omega_{2}\right]=\int_{f_{1}}^{f_{2}} P(\omega, t) d \omega
$$

Each HI represents a subtype of blood vessel or different regions in the vessels, according to the blood flow shear rate. ${ }^{9}$ It is possible to distinguish between large vessels such as arteries and arterioles and small vessels such as capillaries or venules, for instance, by observing a pulsatile pattern resembling the blood pressure wave in HIs that are associated with pulsatile blood vessels ${ }^{8}$ (see also Figure 1 ).

$\mathrm{HI}$ is an absolute parameter that may vary between trials due to slight changes in sensor location or proximity to the skin. The relative $\mathrm{HI}$ (relHI) is a normalized parameter defined as: ${ }^{8}$ 


$$
\operatorname{relHI}\left(\left[f_{1}, f_{2}\right], t\right)=\frac{H I\left[f_{1}, f_{2}\right]}{H I[0, f \operatorname{samp}]}
$$

where $\mathrm{f}_{\text {samp }}$ is the sampling frequency of the measured signal. The variations in relHI following physiological events can be compared between different measurements.

An additional parameter that is extracted from the mDLS signal is the relative blood flow velocity. This parameter is equivalent to the value measured by laser Doppler flowmetry, ${ }^{14}$ which is used in various applications of hemodynamic research, including quantification of acute noxious stimuli. ${ }^{12}$ In Doppler flowmetry, the measured Doppler-evoked frequency shift is proportional to the particles' velocity and thus the statistical representation of the skin blood velocity can be derived. Formally, the equivalent skin blood velocity parameter can be defined as the normalized first moment of the power spectrum of the mDLS sensor signal. We term this parameter the relative blood velocity (RBV).

$$
R B V=\int \omega P(\omega, t) d \omega / \int P(\omega, t) d \omega
$$

Our goal was to determine the significance of the various hemodynamic parameters in relation to noxious stimulation. To this end, we tested the responses of two hemodynamic parameters derived from the optical signal of the mDLS sensor: relHI and RBV. These hemodynamic parameters are directly related to autonomic nervous system activity, ${ }^{10}$ and could potentially be used for detecting (and quantifying) the autonomic response to nociceptive events.

\section{MATERIALS AND METHODS}

\subsection{Measurement system}

Two mDLS sensors (Elfi-Tech Ltd., Rehovot, Israel), each of which contain a probe and a three-axis accelerometer (Fig. 1), are positioned on the skin and gently fixated with adhesive tape. One sensor is placed on the palmar aspect of the left index finger, the other on the palmar aspect of the right index finger. The probes are made up of $850 \mathrm{~nm}$ vertical cavity surface emitting laser operating in CW mode and two detectors. The mDLS sensor placed on the skin projects a laser beam at blood vessels in the dermis. Light scattered from passing red blood cells (RBCs) in superficial blood vessels is collected by the photo detectors (Fig. 1A). The inputs of the accelerometers are utilized for identifying and removing motion artifacts. The sensors are connected to an electronic control unit (Elfor-1, Elfi-Tech Ltd.) that collects the data at $48 \mathrm{kHz}$ using a computer interface program.

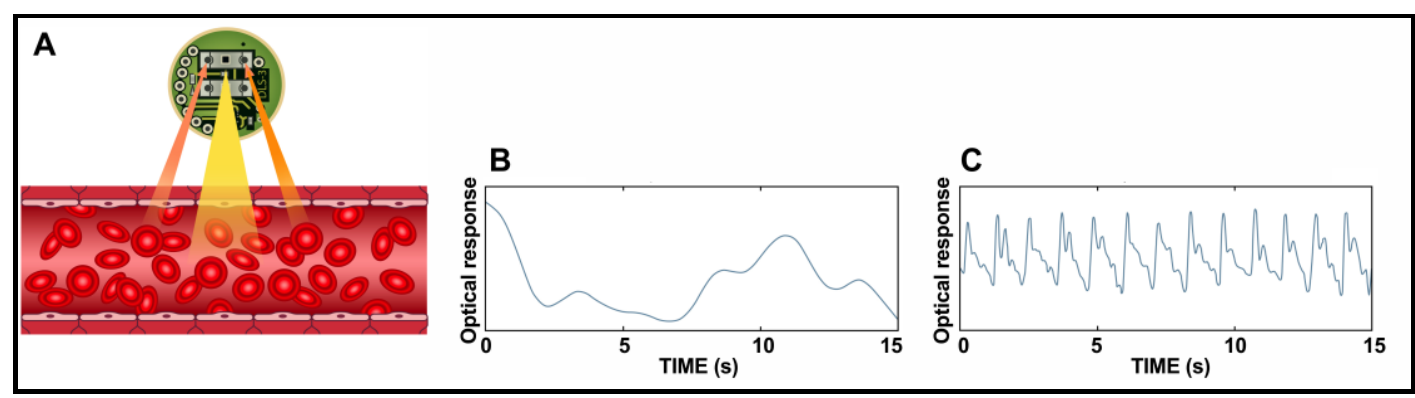

Fig. 1 Schematic overview of the miniature dynamic light scattering (mDLS) technique used in this study. The small (diameter $1 \mathrm{~cm})$ mDLS sensor radiates laser light through the skin. The light reflected from flowing red blood cells is collected via two optical sensors (A) and further analyzed. Non-pulsatile (B) and pulsatile (C) signal are derived from the fluctuations in intensity of the reflected optical signal through power spectrum analysis.

\subsection{Subjects}

The protocol, with reference code P15.156, was performed after obtaining approval from the local Human Ethics Committee (Commissie Medische Ethiek, Leiden University Medical Center, Leiden, The Netherlands) and was registered at www.trialregister.nl under number 5454. All subjects gave oral and written informed consent before enrolment into the study. The data from the 17 healthy subjects (10 women/7 men; age $23.5 \pm 3.4$ years, range: 19-31 years; body mass index $22.5 \pm 1.8 \mathrm{~kg} / \mathrm{m}^{2}$, range $19.0-26.3 \mathrm{~kg} / \mathrm{m}^{2}$ ) are presented in this study. 


\subsection{Stimulation protocol}

First, subjects underwent electrical and heat pain tests. We first determined the thresholds to pain detection (pain detection threshold, Pth) and to pain tolerance (Ptol) for both tests; Pth was defined as NRS $=1$, Ptol as NRS $=10$, with just integers allowed for scoring.

Electrical pain was induced with a computer interfaced current stimulator (CICS, Leiden University Medical Center, Leiden, The Netherlands). ${ }^{13}$ The stimuli were applied to the skin overlying the left shinbone, through two surface electrodes (electrode surface area $0.8 \mathrm{~cm}^{2}$; space between the electrodes $2 \mathrm{~cm}$ ). For detection of Pth and Ptol, an escalating current (5-s trains of $200 \mathrm{~ms}$ pulses at $10 \mathrm{~Hz}$ ) was given from 0 to $128 \mathrm{~mA}$ at a rate of $0.5 \mathrm{~mA} / \mathrm{s}$, during which the subjects indicated their Pth and Ptol by flipping a switch. This process was repeated at least 3 times to obtain an average value $\pm 0.5 \mathrm{~mA}$ for both Pth and Ptol.

Heat pain was induced through a $3-\mathrm{cm}^{2}$ thermode positioned on the volar side of the non-dominant forearm. The thermode was connected to the Pathway Neurosensory Analyzer (Medoc Ltd, Ramat Yishai, Israel), which controls the temperature of the thermode. To determine the temperatures that result in Pth and Ptol we randomly delivered ten to fifteen 30-s heat stimuli with fixed temperatures in the range of 40.0 to $47.9^{\circ} \mathrm{C}$. The subjects scored the NRS of each stimulus; the lowest temperature with NRS scores 1 and 10 were considered Pth and Ptol, respectively. This process was repeated until reproducible values were obtained (i.e. $\pm 0.5^{\circ} \mathrm{C}$ ).

After obtaining Pth and Ptol values for electrical and heat tests, we constructed a linear distribution of 8 interpolated currents and temperatures in between Pth and Ptol, corresponding with estimated NRS scores of 2 to $9 .{ }^{11,12}$ We then randomly applied stimuli to the subjects corresponding with NRS values 1, 4, 6 and 9 with at least 1-min intervals between stimuli. First one complete set of stimuli (heat or electrical) was applied and followed by a second set after a 30-min pause, the order of which was random. Both heat and electrical stimuli lasted 30s. The subjects were blind to the expected NRS values of the stimuli. After each stimulus, the subjects were asked to rate the stimulus using the NRS.

\subsection{Data Analysis}

We used the hemodynamic information from the mDLS sensor to assess whether this new approach can detect nociceptive responses during application of a series of thermal and electrical nociceptive stimuli in awake healthy volunteers. Two frequency bands were selected for calculating the relHI. The first was adjusted to represent the relative blood flow for very small vessels, such as capillaries or venules (spectrum band of 0-500 Hz) and the second band was selected to represent the periodically oscillating high shear rates for greater vessels, such as arterioles $(4-10 \mathrm{kHz})$; the appearance of the pulsatile component was used as a marker to ensure that the latter HI represents the blood flow of the arterioles or small arteries. From here on these HI parameters will be referred to as the small vessels representation (SVR) and the large vessels representation (LVR).

For data analysis, the data was divided into two main groups, electrical and heat pain stimulation. Each group was further divided into four subgroups referring to the different pain intensities applied at (expected) NRS scores of 1, 4, 6 and 9 (NRS 1, NRS 4, NRS 6 and NRS 9). The differences between the responses and baseline values were calculated for each of the mDLS derived measures (HR, RBV and relHI). Baseline refers to a 60-s period of relaxation prior to any stimulus given; the stimulus refers to the 30-s mean of the response. To get an indication of the temporal profile of the response, we additionally divided the response into three 10-s episodes $(0-10 \mathrm{~s}, 10-20 \mathrm{~s}$ and 20-30 s) and calculated their differences with baseline values. The data was analyzed using paired-t-tests with p-values $<0.005$ considered significant.

\section{RESULTS}

\subsection{HR and RBV response to pain stimuli}

Relative to baseline, no significant changes in HR were observed during stimulation in heat or electrical tests (Table 1). 


\begin{tabular}{|c|c|c|c|c|c|}
\hline \multicolumn{3}{|c|}{$\Delta \mathrm{HR}(\mathrm{BPM})$ (electric) } & \multicolumn{3}{|c|}{$\Delta \mathrm{HR}$ (BPM) (heat) } \\
\hline $0-10 s$ & $10-20 \mathrm{~s}$ & $20-30 \mathrm{~s}$ & $0-10 s$ & $10-20 \mathrm{~s}$ & $20-30 \mathrm{~s}$ \\
\hline $0.93 \pm 3.48$ & $4.51 \pm 6.35$ & $2.67 \pm 7.39$ & $1.17 \pm 3.85$ & $-0.07 \pm 4.1$ & $0 \pm 4.87$ \\
\hline$(P=0.3)$ & $(P=0.01)$ & $(P=0.2)$ & $(P=0.2)$ & $(P=0.9)$ & $(P=0.99)$ \\
\hline \multicolumn{3}{|c|}{$\Delta R B V \times 10^{3}\left(\mathrm{~Hz}^{-1}\right)$ (electric) } & \multicolumn{3}{|c|}{$\Delta \operatorname{RBV} \times 10^{3}\left(\mathrm{~Hz}^{-1}\right)$ (heat) } \\
\hline $0-10 s$ & $10-20 \mathrm{~s}$ & $20-30 s$ & $0-10 s$ & $10-20 \mathrm{~s}$ & $20-30 \mathrm{~s}$ \\
\hline$-2.99 \pm 3.69$ & $-2.54 \pm 3.5$ & $-2.87 \pm 2.4$ & $-1.01 \pm 2.81$ & $-1.56 \pm 4.36$ & $-1.96 \pm 4.37$ \\
\hline$(P=0.005)$ & $(P=0.01)$ & $(P<0.0005)$ & $(P=0.2)$ & $(P=0.2)$ & $(P=0.1)$ \\
\hline
\end{tabular}

Table 1. Effect of noxious stimulation on heart rate (HR) and Doppler equivalent (DE). $\Delta H R$ is the change in heart rate from baseline; $\triangle \mathrm{DE}$ is the change in Doppler equivalent from baseline. The data are the mean values $\pm \mathrm{SD}$ measured during the 30 -s stimulation in response to stimulation at NRS9.

However, a decrease in RBV values was observed for both types of stimulation (Table 1, Figure 2).
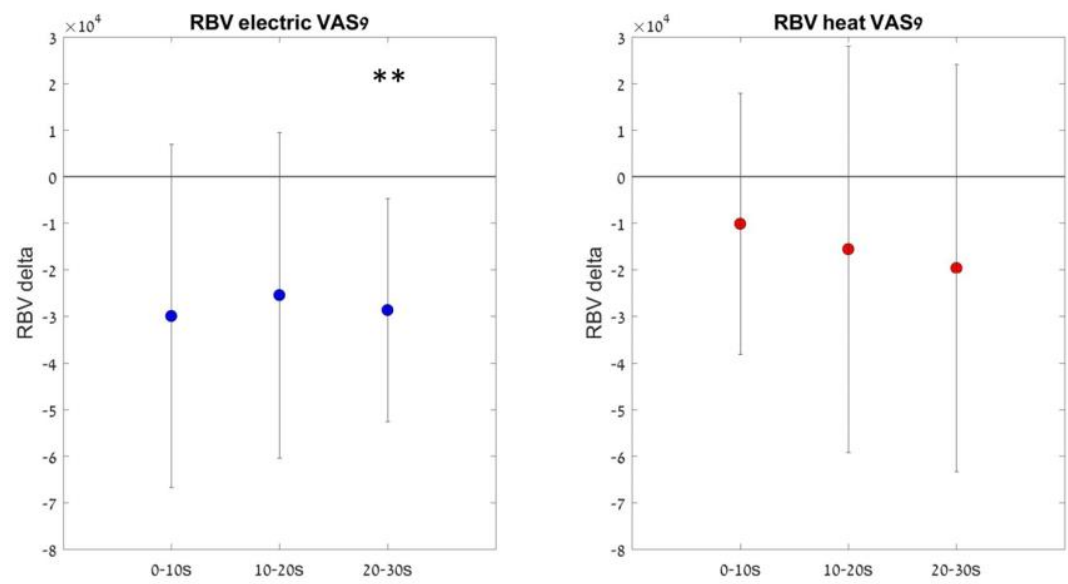

Fig. 2 Temporal profile of the change in relative blood velocity (RBV) during electrical (A) and heat (B) stimulation at NRS9. The data (from the left finger) are divided into three time windows, 0-10 s, 10-20 s and 20-s, of stimulus time. Values are mean \pm SD. $* * p$ $<0.0005$. RBV in arbitrary units.

\subsection{Relative hemodynamic index response}

Examples of relHI responses obtained in one subject on the left and right index fingers during electrical stimulation at NRS 9 are given in Figure 3. During stimulation, an increase in relative flow of the small non-pulsatile vessels (SVR) and a decrease in relative flow of the larger pulsatile vessels (LVR) was observed with a rapid return towards baseline values after termination of the stimulus. The responses of the left and right index fingers were highly correlated with identical directions of effect in $90 \%$ for electrical stimuli and $84 \%$ for heat stimuli. This is indicative of a systemic effect of noxious stimulation on skin hemodynamics. 


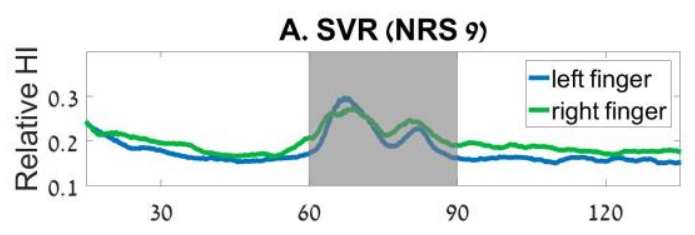

B. LVR (NRS 9)

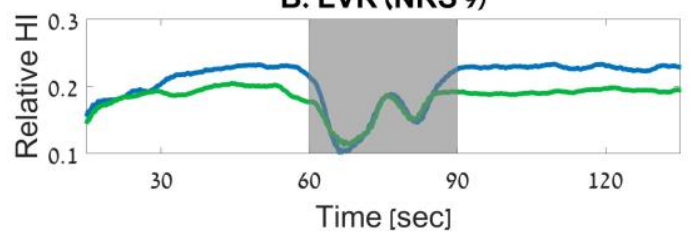

Fig. 3 Example of the effect of electrical noxious stimulation at a numerical rating score (NRS) of 9 on relative hemodynamic index (HI) of the small vessel representation (SVR; A) and large vessel representation (LVR; B) of one subject. The responses of the left finger (blue lines) and right finger (orange lines) are depicted. The grey bar indicates the period of electrical stimulation. HI in arbitrary units.

The effect of electrical and heat stimulation on relative blood flow in the small and large vessel representations are demonstrated in figure 4. At all stimulus intensities, there was an increase in the relative blood flow in the SVR and a decrease in relative blood flow in the LVR. Figure 4 depicts the change in relHI responses for electrical and heat pain stimuli for NRS9 (data from the left finger).
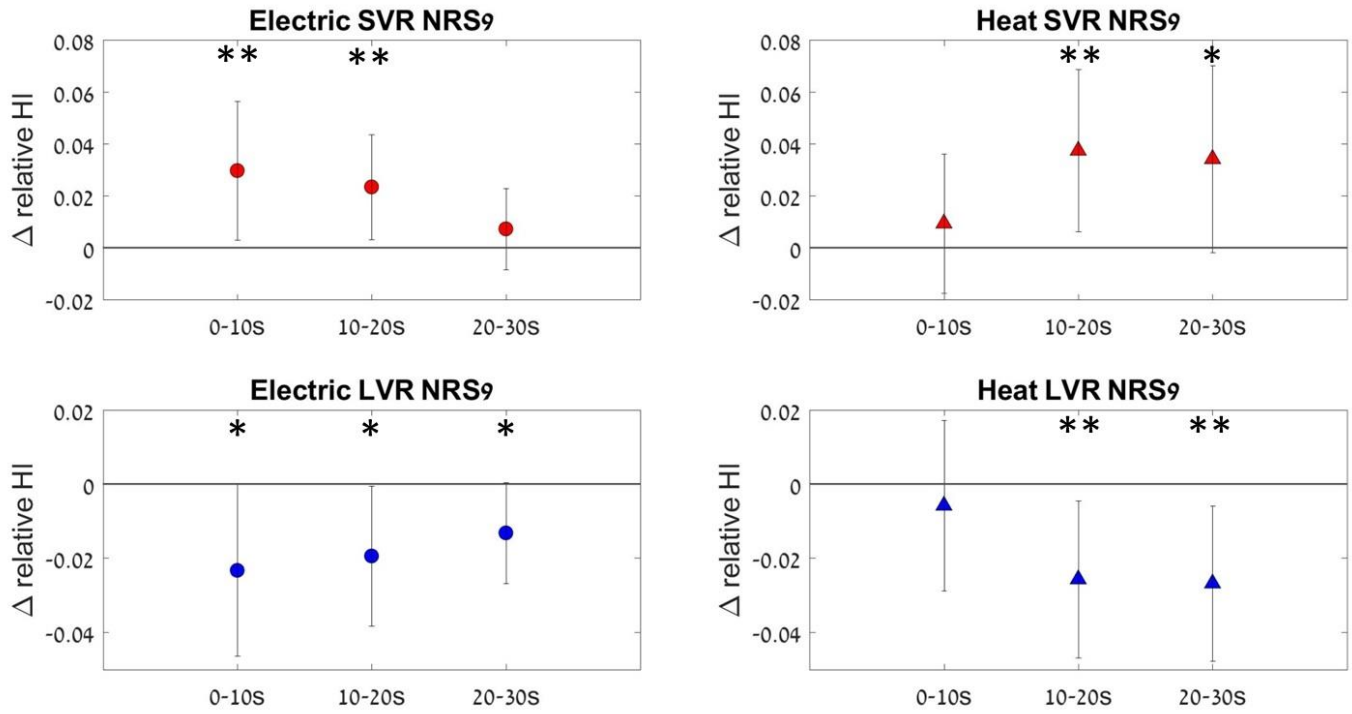

Fig. 4 Temporal profile of the change in relative hemodynamic index (HI) during electrical and heat stimulation at different stimulus intensities. A, B. Responses for the small vessel representative (SVR). C, D. Responses for the large vessel representative (LVR). The data are divided into three time windows, $0-10 \mathrm{~s}, 10-20 \mathrm{~s}$ and $20-\mathrm{s}$, of stimulus time. Values are mean $\pm \mathrm{SD}$. $* \mathrm{p}<0.005, * * \mathrm{p}<$ 0.0005 . HI in arbitrary units.

For electrical stimulation, the change in both SVR and LVR is immediate, occurring in the first 10-s episode, followed a by a slow decline towards baseline. The changes in relHI responses for heat pain stimuli are somewhat slower in onset and offset and less marked with a peak in response occurring in the 10-20 s episode.

To get an indication of the stimulus intensity- $\Delta$ relHI relationship, we plotted the reported NRS scores against $\Delta$ relHI (obtained in the 20-30 s stimulus period) in Figure 5. 

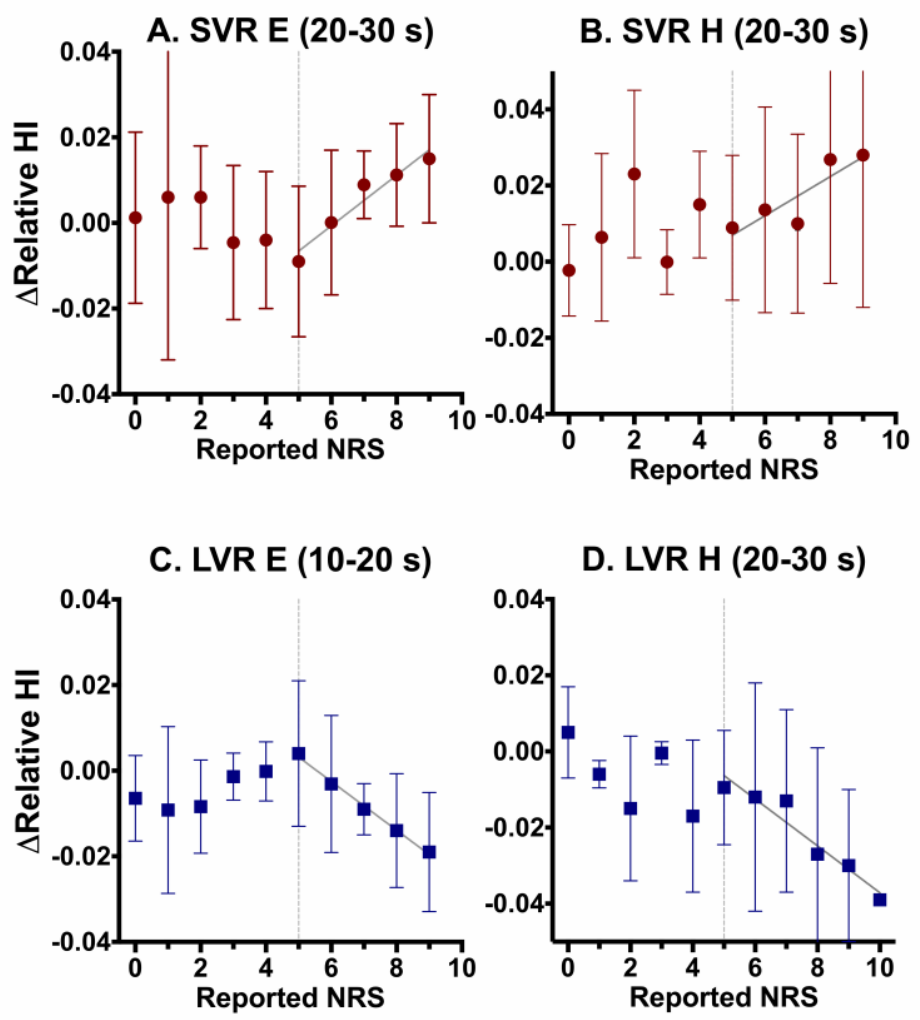

Fig. 5 Effect of reported numerical rating scores (NRS) on the change in relative hemodynamic index ( $\Delta$ relative HI) for the small vessel representative (SVR, panels $\mathbf{A}$ and $\mathbf{B}$ ) and the large vessel representative (LVR, panels $\mathbf{C}$ and $\mathbf{D}$ ) for noxious electrical (E, panels $\mathbf{A}$ and $\mathbf{C}$ ) and noxious heat (H, panels $\mathbf{B}$ and $\mathbf{D})$. A linear regression line was drawn for NRS values $\geq 5$. Data are the mean $\Delta$ relative HI values \pm SD obtained from the 20-30 s of the noxious stimulus. HI in arbitrary units. In panel B one subject reported an NRS of 10, his $\Delta$ relative HI was 0.15 and not included in the figure or in the linear regression analysis.

We observed a linear intensity-response relationship for NRS values $\geq 5$ for both stimuli in the SVR and LVR. The dose dependency was more robust for noxious electrical pain stimuli than for noxious heat stimuli.

\section{DISCUSSION}

In the current study, we used hemodynamic and relative hemodynamic indices, derived from scattered light intensity analysis of moving red blood cells in the vessels of the skin, to detect physiological responses to acute noxious cutaneous stimulation. Skin blood flow dynamics were measured with the mDLS sensor during the application of 30-s electrical and thermal stimuli of varying intensities in a group of young healthy volunteers. The main findings of our study are that (1) the mDLS sensor was able to detect noxious events as measured by relHI; (2) a linear dose relationship between stimulus intensity and the change in relHI relative to baseline was observed for reported NRS scores > 5; (3) heart rate was unable to detect the noxious stimuli at the intensities applied in this study; (4) there is a decrease in blood flow velocity (as quantified by the Doppler equivalent); (5) an inverse response to noxious stimulation was observed in the LVR and the SVR, with a reduction in relative flow in pulsatile vessels of the skin and an increase in relative flow in non-pulsatile vessels; (6) changes in relative hemodynamic index occurred simultaneously in left and right index fingers, independent of the site of stimulation.

In our study, two different representations of the hemodynamics of blood vessels of the skin were extracted from the scattered light intensity pattern, one which represents red blood cell flow in SVR and one which represents red blood cell flow in LVR. Similar approaches to blood flow hemodynamics are increasingly used in biomedical research. For example, in rats, this technique has been applied to quantify anastomotic healing in colorectal surgery. ${ }^{15}$ In this study, it was shown that non-pulsatile (e.g. capillary) anastomotic perfusion is a useful marker of anastomotic leakage in a rat 
colectomy model. Additionally, in a group of 19 volunteers, the effect of mental stress on the hemodynamic index was tested using the mDLS sensor showing large and consistent effects of stress on hemodynamic changes in the SVR. ${ }^{9}$

In the current study, apart from flow-related parameters, we extracted two "standard" hemodynamic markers, heart rate and RBV from the mDLS sensor response to nociceptive events. We observed no significant response of HR, in agreement with earlier studies. ${ }^{16}$ In contrast, flow-related parameters such as RBV and relative blood flow in the LVR and SVR, showed significant changes to nociceptive stimuli. We relate the noxious stimulation-induced reduction in skin perfusion to vasoconstriction of the larger blood vessels (LVR: arterioles, small arteries) of the skin secondary to autonomic nervous system activation. ${ }^{19,20}$ This response is most probably neurogenic, i.e. due to alpha-adrenergic receptor activation secondary to epinephrine and norepinephrine release from sympathetic nerves that innervate skin arterioles and arteries. A humoral component seems unlikely, given the temporal profile of the observed response (rapid onset and offset of hemodynamic changes; Fig. 4C-D). Whether the vasoconstriction was restricted to the pulsatile vessels of the skin remains unknown, but the central sympathetic response may also have effects at sites other than the skin.

Combining the response to noxious stimuli of flow-related indices, we postulate that there is a decrease in total flow to the LVR combined with a redistribution of blood flow between the LVR and the SVR. The analysis of absolute HI values (data not shown) indicates that the increase in flow of the SVR is relatively minor compared to the decrease in flow of the LVR. The observation that changes in the LVR and SVR exhibit similar dynamics (Fig. 3) suggests that the mechanisms of flow changes in pulsatile and non-pulsatile vessels are tightly coupled. It may be that the increase in $\Delta$ relHI of the SVR is related to redistribution of flow. This is possibly due to a local dynamic autoregulatory or reflectory response independent of neural control (e.g., through the release of gaseous signaling molecule and potent vasodilator nitric oxide from capillary and venular endothelial cells), and/or secondary to sympathetic-fiber release of vasodilators. ${ }^{21,22}$

The temporal profile of the relHI responses to electrical and heat stimulation differed in their dynamics (Fig. 4). Relative to the response to electrical stimuli that peaked early (within the first 10-s period of the stimulus), the response to heat stimulation was slower, with a peak response in the middle one-third of the stimulus. Moreover, more robust changes in response to electrical stimulation were observed compared to heat stimulation (Fig. 5). As discussed previously, ${ }^{11}$ different pain models activate different pain pathways with differences in central processing.

The decrease in RBV for both types of stimulation is in agreement with the dynamics of the relative blood flow in SVR and LVR - a decrease in RBV indicates a shift in energy towards lower frequency bands. Although the decrease was statistically significant only for electrical stimulation, we postulate that the delayed onset of the response to heat stimulation may lead to a significant decrease in RBV at a slightly later time period which we cannot observe using the current protocol.

Interestingly, we observed a linear stimulus intensity- $\Delta$ relHI response relationship at reported NRS values $>5$ (Fig. 5). This suggests that just reported stimuli at NRS intensities greater than 5 were perceived as painful enough to cause a significant autonomic response. However, somewhat to our surprise, even at the lowest intensity electrical stimulus, i.e. NRS 1, corresponding to the first perception of pain (pain threshold), a similar trend in the hemodynamic response was observed. Since our study was performed in awake subjects, apart from the central processing of the afferent nociceptive input, some emotional or stress-related effects may have contributed to the hemodynamic responses we observed. Further studies are still needed to assess the response to pain under conditions in which conscious processing is absent or reduced.

To summarize, we applied the novel technique of dynamic light scattering to determine the effect of noxious stimulation on hemodynamic parameters in awake volunteers. While parameters that are commonly used such as HR were not able to detect noxious events, we observed that mDLS parameters such as RBV and relative blood flow could detect nociceptive stimuli and consequently could serve as objective biomarkers of nociception (acute pain). Moreover, these biomarkers provided some insight into the physical and physiological changes in hemodynamics that occur during noxious stimulation. Additional studies should address the ability of the mDLS sensor in detecting noxious stimuli during anesthesia or deep sedation and determine whether combining the hemodynamic parameters into one index would further increase the ability of the system to detect noxious events. 


\section{DISCLOSURES}

Adi Schejter Bar-Noam, Ilya Fine and Louis Shenkman are employees of Elfi-Tech Ltd., Rehovot, Israel. All other authors declare no competing interests.

\section{REFERENCES}

1. C. Martini et al., "Ability of the Nociception Level, a Multiparameter Composite of Autonomic Signals, to Detect Noxious Stimuli during Propofol-Remifentanil Anesthesia," Anesthesiology: The Journal of the American Society of Anesthesiologists 123 (3), 524-534 (2015).

2. M. Larson et al., "Alfentanil blocks reflex pupillary dilation in response to noxious stimulation but does not diminish the light reflex," Anesthesiology: The Journal of the American Society of Anesthesiologists 87 (4), 849-855 (1997).

3. O. Shimoda et al., "Skin vasomotor reflex predicts circulatory responses to laryngoscopy and intubation," Anesthesiology: The Journal of the American Society of Anesthesiologists 88 (2), 297-304 (1998).

4. X. Chen et al., "Comparison of Surgical Stress Index-guided Analgesia with Standard Clinical Practice during Routine General AnesthesiaA Pilot Study," Anesthesiology: The Journal of the American Society of Anesthesiologists 112 (5), 1175-1183 (2010).

5. M. Rantanen et al., "Novel multiparameter approach for measurement of nociception at skin incision during general anaesthesia," British journal of anaesthesia 96 (3), 367-376 (2006).

6. T Ledowski et al., "Surgical pleth index: prediction of postoperative pain and influence of arousal," British journal of anaesthesia 117 (3), 371-374 (2016).

7. WI Goldburg, "Dynamic light scattering," American Journal of Physics 67 (12), 1152-1160 (1999).

8. I. Fine et al., "A non-invasive method for the assessment of hemostasis in vivo by using dynamic light scattering," Laser Physics 22 (2), 469-475 (2012).

9. I. Fine et al., "A new sensor for stress measurement based on blood flow fluctuations." In Dynamics and Fluctuations in Biomedical Photonics XIII, vol. 9707, p. 970705. International Society for Optics and Photonics, (2016). (http://dx.doi.org/10.1117/12.2212866)

10. L. Bernardi et al., "Synchronous and baroceptor-sensitive oscillations in skin microcirculation: evidence for central autonomic control," American Journal of Physiology-Heart and Circulatory Physiology 273 (4), H1867H1878 (1997).

11. L. Oudejans et al., "Translation of random painful stimuli into numerical responses in fibromyalgia and perioperative patients," Pain 157 (1), 128-136 (2016).

12. B. Torensma et al., "Pain sensitivity and pain scoring in patients with morbid obesity," Surgery for Obesity and Related Diseases 13 (5), 788-795 (2017).

13. E. Olofsen et al., "Alfentanil and Placebo Analgesia: No Sex Differences Detected in Models of Experimental Pain," Anesthesiology: The Journal of the American Society of Anesthesiologists 103 (1), 130-139 (2005).

14. C. Limjeerajarus, "Laser Doppler flowmetry: basic principle, current clinical and research applications in dentistry," Chulalongkorn University Dental Journal 37 (1), 123-136 (2014).

15. Z. Wu et al., "Postoperative hemodynamic index measurement with miniaturized dynamic light scattering predicts colorectal anastomotic healing," Surgical innovation 23 (2), 115-123 (2016).

16. P. Ling et al., "Assessment of postoperative pain intensity by using photoplethysmography," Journal of anesthesia 28 (6), 846-853 (2014).

17. P. Shi et al., "Serial assessment of laser Doppler flow during acute pain crises in sickle cell disease," Blood Cells, Molecules, and Diseases 53 (4), 277-282 (2014).

18. E. Sarton et al., "Acute pain and central nervous system arousal do not restore impaired hypoxic ventilatory response during sevoflurane sedation," Anesthesiology: The Journal of the American Society of Anesthesiologists 85 (2), 295-303 (1996).

19. B. G. Wallin, "Neural control of human skin blood flow," Journal of the autonomic nervous system 30, S185S190 (1990). 
20. Y. Ootsuka and Mutsumi Tanaka, "Control of cutaneous blood flow by central nervous system," Temperature 2 (3), 392-405 (2015).

21. T. E. Wilson et al., "Dynamic autoregulation of cutaneous circulation: differential control in glabrous versus nonglabrous skin," American Journal of Physiology-Heart and Circulatory Physiology 289 (1), H385-H391 (2005).

22. Joseph Loscalzo, "The identification of nitric oxide as endothelium-derived relaxing factor," Circulation research 113 (2), 100-103 (2013). 